This is an electronic reprint of the original article. This reprint may differ from the original in pagination and typographic detail.

Author(s): Hartikainen, Markus; Lovison, Alberto

Title: $\quad$ PAINT-SiCon: constructing consistent parametric representations of Pareto sets in nonconvex multiobjective optimization

Year: $\quad 2015$

Version:

Please cite the original version:

Hartikainen, M., \& Lovison, A. (2015). PAINT-SiCon: constructing consistent parametric representations of Pareto sets in nonconvex multiobjective optimization. Journal of Global Optimization, 62(2), 243-261. https://doi.org/10.1007/s10898-0140232-9

All material supplied via JYX is protected by copyright and other intellectual property rights, and duplication or sale of all or part of any of the repository collections is not permitted, except that material may be duplicated by you for your research use or educational purposes in electronic or print form. You must obtain permission for any other use. Electronic or print copies may not be offered, whether for sale or otherwise to anyone who is not an authorised user. 


\title{
PAINT-SiCon: Constructing Consistent Parametric Representations of Pareto Sets in Nonconvex Multiobjective Optimization
}

\author{
Markus E. Hartikainen · Alberto Lovison
}

Received: date / Accepted: date

\begin{abstract}
We introduce a novel approximation method for multiobjective optimization problems called PAINT-SiCon. The method can construct consistent parametric representations of Pareto sets, especially for nonconvex problems, by interpolating between nondominated solutions of a given sampling both in the decision and objective space. The proposed method is especially advantageous in computationally expensive cases, since the parametric representation of the Pareto set can be used as an inexpensive surrogate for the original problem during the decision making process.
\end{abstract}

Keywords Multiple criteria programming · Nonlinear programming · Piecewise linear approximation

\section{Introduction}

Multiobjective optimization concerns simultaneously optimizing multiple conflicting objectives (see, e.g., [1, 2]). Multiobjective optimization has its roots in economics (see [3]). However, many effective applications can be nowadays found, among others, in mechanics, aerospace and automotive engineering, environmental science, medicine and even biology $([4-8])$. For a typical multiobjective optimization problem there exist multiple Pareto optimal solutions, i.e., solutions where

Markus E. Hartikainen

Department of Mathematical Information Technology,

University of Jyväskylä,

PO box 35 (Agora),

FI-40014 University of Jyväskylä

Finland,

E-mail: markus.e.hartikainen@jyu.fi

Alberto Lovison

Dipartimento di Matematica,

Università degli Studi di Padova,

Via Trieste, 63

35121-I Padova,

Italy,

E-mail: lovison@math.unipd.it 
none of the objectives can be improved without impairing at least one of the remaining objectives. However, in most cases, only one solution has to be selected for implementation. The person, or the group, appointed for choosing such a solution is called the decision maker. It is often assumed that the decision maker chooses the solution through an enlightened screening of the range of the available values of the objective functions. Naturally, a better decision can be taken when the decision maker is made aware of an as large as possible set of alternative Pareto optimal solutions.

To this purpose, Pareto front and Pareto set approximation methods become advantageous. Following [9], we mean by a Pareto front approximation a set in the objective space that can be used as a surrogate for the actual Pareto front in decision making. A Pareto set approximation is a similar approximation in the decision space. Several approximation methods can be found in the literature (see, among many others, [9-12]), but only few of them are effective for the application to nonconvex problems and even fewer involve the surrogate set in the actual decision making process (see, e.g., $[13,14]$ ).

A subclass of the Pareto set approximation methods consists of the so-called continuation methods, which translate mathematically the intuition of continuity and regularity that is apparent to human eye even when visualizing also a relatively small set of Pareto optimal solutions. These methods, rather than generate an as large as possible set of Pareto optimal solutions, attempt to trace explicitly the Pareto curves and surfaces. Continuation can be performed either locally or globally. Local continuation consists in considering a given Pareto optimal solution $x^{0}$ and then attempt to trace a mesh of new Pareto optimal solutions in a small neighborhood of $x^{0}$. Iterating this process starting from one of the newly found solutions, one can generate the local branch of the Pareto set passing through $x^{0}$ (see $[11,15-17]$, and the references therein). Global continuation, on the other hand, is intended to draw consistently connections among a given set of optimal solutions, in order to build a higher-dimensional structure, which is usually a simplicial complex (see [18]), globally approximating the Pareto set. These ideas are reminiscent of "connecting the dots" children's games. Alternatively, a global continuation could start from a geometrical decomposition of the feasible set (e.g., a Delaunay tessellation, a cubic tessellation) and next produce a linear approximation of the portion of the Pareto set passing through each tessellation cell $[12,19,20]$. The strengths and weaknesses of these methods emerge when dealing with highly nonlinear and nonconvex problems, where the Pareto front can be disconnected, or composed by different local branches intersecting one another, as illustrated in Figure 1.

By keeping these problems in mind, we have developed a new PAINT-SiCon global continuation method. The input of the PAINT-SiCon algorithm is a sampling of the decision space. The algorithm interpolates between the nondominated points of the sample both in the decision and in the objective space, respecting the domination structure of the objective space. Here, by respecting the domination structure, we mean that the constructed set is inherently nondominated as defined in [14]. Furthermore, the method detects connected components of the set of Pareto optimal solutions. The advantages of the method include that it is parameter free and especially developed to deal with nonconvex problems. Finally, the parametric representation of the approximation is computationally inexpensive to handle and 

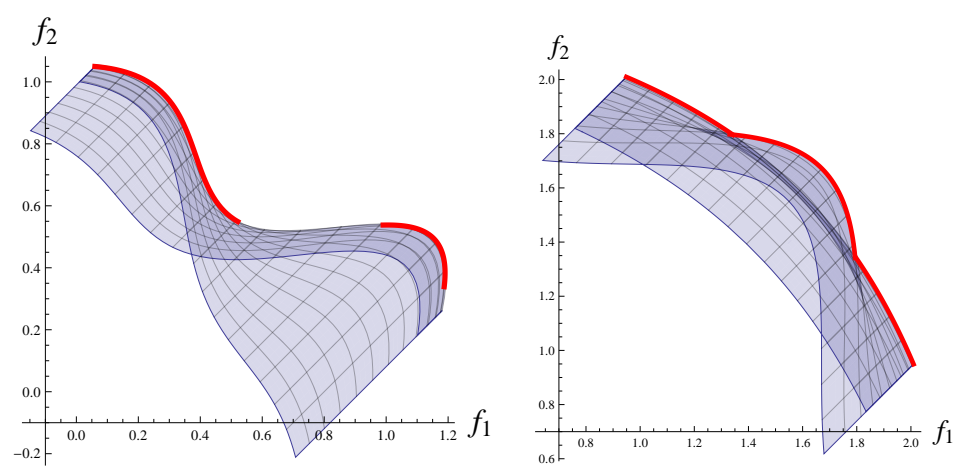

Fig. 1: Typical problems emerging with nonconvex objective functions: on the left, the Pareto front is disconnected; and, on the right, the Pareto front is composed by two distinct branches, superimposing one to the other.

can be viewed as a surrogate problem, like e.g., in [21]. Thus, the method facilitates decision making by embedding the use of the approximation in any interactive multiobjective optimization method.

The algorithm proposed in this paper draws ideas from two Pareto set approximation algorithms, i.e., PAINT [13, 21] and SiCon [19]. The PAINT method differs from the new PAINT-SiCon in the fact that the former works only in the objective space, producing an interpolation of the Pareto front consistent with the dominance structure (see Section 2.2 for more details). The other forebear method, SiCon, uses a tessellation in the decision space and local optimality conditions for tracing piece--wise linear approximation of the Pareto set and of the Pareto front (see Section 2.3 for more details). However, both of these methods have weaknesses: PAINT does not consider the functional relation between the decision space and the objective space and, thus, does not give any information about values of the decision variables. On the other hand, SiCon produces an approximation of all the local Pareto optimal solutions, which could possibly include some dominated points. In addition, SiCon requires the computation of the whole Delaunay tessellation of the sample points and derivative information on the objective functions and this, for high-dimensional problems, can be computationally exhaustive. The PAINT-SiCon algorithm proposed in this paper attempts to combine the strengths of both algorithms while avoiding their respective shortcomings. Especially, the new PAINT-SiCon method will be able to approximate both in the objective and decision spaces, detect disconnectedness in the Pareto set and is computationally less expensive than the SiCon method. In addition, the new PAINT-SiCon method includes a new way of including the knowledge about the constraints of the problem into the construction of the approximation, which is something that neither forebear method can do.

This paper is organized as follows: First, in Section 2 we recall relevant mathematical background, fix the notation and give an overview of the two forebear methods PAINT and SiCon (concentrating on details about their strengths and weaknesses). In addition, the paper contains an electronic supplementary material, where we review terminology related to computational and differential geometry 
that is necessary for this paper, but is not often discussed in multiobjective optimization literature. In Section 3, the mathematical basis for the new PAINT-SiCon method is established, while the technical details of the algorithm are given in Section 4. Section 5 describes the workings of the method on two critical nonconvex problems. Conclusions and perspectives are presented in Section 6.

\section{Preliminaries}

In this section, we review the relevant mathematical background from the perspective of this paper and give an overview of the two forebear approximation methods PAINT and SiCon. Further background material, specifically related to computational and differential geometry that are not often discussed in multiobjective optimization literature, is reported in Section 1 of the Supplementary Material (SM). In the Supplementary Material, we review the definitions that are needed in this research and set the notation and terminology. We start with the basic definitions of multiobjective optimization.

\subsection{Multiobjective optimization}

Let $X \subseteq \mathbb{R}^{n}$ and consider a vector function

$$
\begin{aligned}
f: X & \longrightarrow \mathbb{R}^{k}, \\
x & \longmapsto f(x)=\left(f_{1}(x), \ldots, f_{k}(x)\right) .
\end{aligned}
$$

- A general multiobjective optimization problem (MOP) consists in optimizing (maximizing or minimizing) jointly the scalar functions $f_{1}, \ldots, f_{k}: X \longrightarrow \mathbb{R}$ :

$$
\max _{x \in X}\left(f_{1}(x), \ldots, f_{k}(x)\right)
$$

- The domain $X$ of the functions is called the feasible set, while any point $x \in X$ is called a (feasible) solution. The functions $f_{1}, \ldots, f_{k}: X \longrightarrow \mathbb{R}$ are called the objective functions or simply the objectives. The space $\mathbb{R}^{n}$ is called the decision space, while $\mathbb{R}^{k}$ is called the objective space. The vectors $z=f(x) \in \mathbb{R}^{k}$, for any $x \in X$, are called outcomes.

Apart from pathological cases, it is not possible to find a solution which is optimal for all objectives taken separately. On the other hand, adopting a natural partial ordering on the objective space $\mathbb{R}^{k}$, it is usually possible to find infinite maximal elements, which in this context are named Pareto optimal solutions.

- A solution $x \in X$ is said to dominate another solution $y \in X$ if $f_{i}(x) \geqslant f_{i}(y)$ for all $i=1, \ldots, k$, and $f_{j}(x)>f_{j}(y)$ for at least one $j \in\{1, \ldots, k\}$. The relation of domination induces a partial ordering on $X$.

- A solution $x^{\star} \in X$ is said Pareto optimal if there does not exist any solution which dominates $x^{\star}$. The image $f\left(x^{\star}\right)$ of a Pareto optimal solution $x^{\star}$ is called a Pareto optimal outcome.

- The set of Pareto optimal solutions is called the Pareto set (PS), while the set of Pareto optimal outcomes is called the Pareto front (PF). Therefore PS is a subset of the decision space $\mathbb{R}^{n}$ and of the feasible set $X$, while PF is a subset of the objective space $\mathbb{R}^{k}$. 


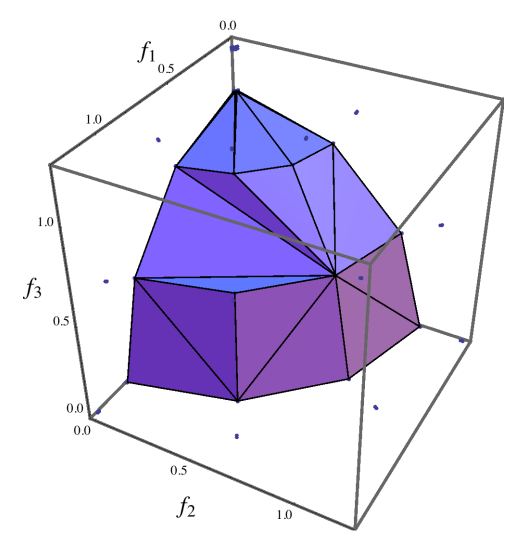

Fig. 2: Application of PAINT to DTLZ2 problem.

\subsection{PAINT: PAreto front INTerpolation}

PAINT is a continuation method operating in the objective space. The PAINT method was proposed in [21], and it is based on the concept of an inherently nondominated PF approximation introduced in [14] and on the mathematical theory presented in [13]. PAINT defines a polyhedral complex using as nodes a given set of Pareto optimal outcomes, in a way that, by construction, there do not exist two vectors in the whole complex one dominating the other. As a first step, the method produces the full Delaunay tessellation based on the given Pareto optimal outcomes, and defines a basic approximation of the PF as the set of the given solutions. Next the method selects sequentially simplexes from the Delaunay tessellation that can be added to the approximation of the PF without impairing its inherent approximation. The resulting approximation of the PF has been proven accurate for a number of test problems. For instance, figure 2 shows an example of applying the PAINT method to approximate the PF of the standard 3-objective DTLZ2 problem [22]. The dots in the figure are the given input to the PAINT method, and the triangles and the quadrilaterals are the approximation constructed by PAINT. The actual PF of the DTLZ2 problem is the part of the ball surface that is in the positive orthand. In this case, the PAINT method correctly traces the shape of the PF.

The main advantage of PAINT is that the approximation constructed can effectively support the decision making process. The surrogate problem is a multiobjective mixed-integer linear problem, therefore is almost computationally inexpensive and can be solved with any standard method ${ }^{1}$. Solving the approximated problem gives a number of candidate Pareto optimal solutions that can be appraised and compared by the decision maker. The selected approximate solutions can be used as reference points for finding the final preferred solution for the original problem, applying for instance the achievement scalarizing problem [23]. The analysis of the approximation can guide the experimenter for a deeper investigation of

\footnotetext{
1 For a comprehensive survey on well established multiobjective optimization algorithms see [2] and the references therein.
} 


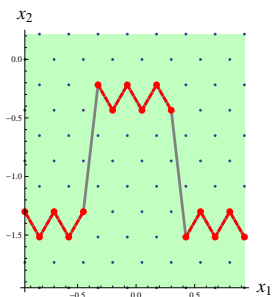

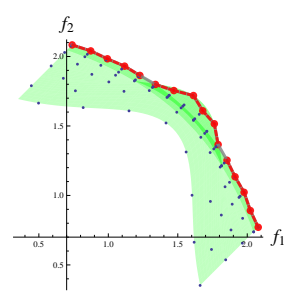

(a)
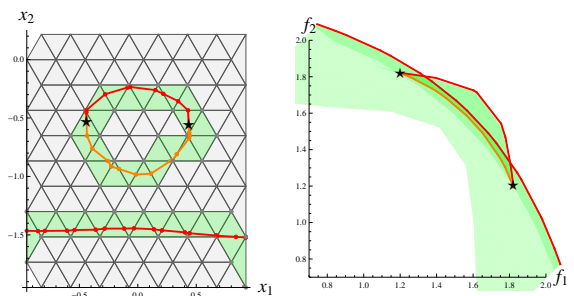

(b)

Fig. 3: (a)Application of the PAINT method to a nonconvex problem. Objective functions are evaluated on a regular grid of points (blue points) and the nondominated set can be extracted (red points). According to the dominance relations PAINT defines connections (i.e., simplexes with those points as vertices) between nondominated outcomes (left panel). These connections can be mapped backwards in the decision space (right panel). Some of these line segments (gray lines) connect solutions which are too far apart, likely belonging to distinct components of the PS. (b) Application of SiCon to the nonconvex two-dimensional problem $L \& H_{2 \times 2}$ (see Section 5). In the left panel, the decision space is shown while the right panel represents the objective space. The red curve is the local PS. Red and orange curves constitute the Pareto critical set.

the problem, involving the computation of brand new Pareto optimal solutions in particularly interesting regions of the PF. Successively the PAINT method can be applied to the augmented set of solutions. ${ }^{2}$

Unfortunately, as already noted in [21], the functional relation between decision variables and the objective values is not involved in the construction of the approximation. Therefore, the behavior of the approximation structure in the decision space can be unpredictable in the presence of nonconvexity. Figure 3(a) illustrates how this can lead the PAINT method to fail to recognize disconnectedness in the PS. The right-side panel in the figure 3(a) shows the outcomes given a sample of points in the objective space (the dots), where the nondominated ones have been given a red color. The red and gray line segments represent the PF approximation constructed by PAINT. However, visualizing the corresponding connecting segments in the decision space, it is clear that some of them (the grey lines) are unreasonable, because their extrema are too far apart. Indeed, such connections have to cross cells which nodes are dominated. Based only on dominance relations in the objectives space, PAINT method has produced a unique connected component for the PS, while clearly the true PS of the problem has three separate components.

Furthermore, although the problem in Figure 3 is an unconstrained problem, there still appears disconnectedness in the PS that PAINT cannot detect. Even more severe issues may emerge if the multiobjective optimization problem is constrained as the PAINT method does not deal with constraints when defining the approximation. We will show in the next section how PAINT-SiCon can cope with constrained problems with disconnected PSs.

2 Further technical details on the PAINT algorithm are given in section 2 of the Supplementary Material (SM). 

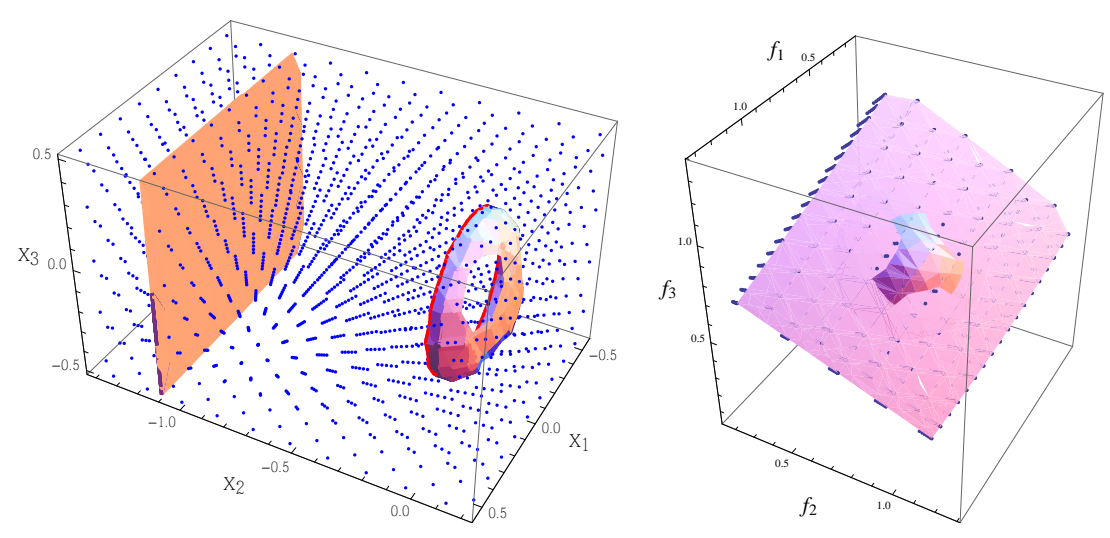

Fig. 4: Application of SiCon on the nonconvex three-dimensional problem $L \& H_{3 \times 3}$ (see Section 5). (Right panel: decision space. Left panel: objective space). The surfaces are an approximation of the set of local Pareto optima.

\subsection{SiCon: Singular Continuation}

Singular Continuation [19, 20], referred to as SiCon, is a method setting forth from the characterization of Pareto optimality based on first and second order derivatives of the objective functions given by S. Smale in [24] (see also [25, 26]) and further using piece-wise linear approximations of implicitly defined manifolds (see, e.g., [27]). According to the SiCon method, linearized algebraic equations of the PS are determined for all the simplexes of a Delaunay tessellation of the feasible set based on a given sample of points. The solution of these equations is a polygon or a polyhedron representing a linear approximation of the part of the PS contained in the tessellation simplex. Taking the union of the simplexes gives a global approximation of the set of local Pareto optimal solutions. The outcome is a quadratically precise approximation of the PS, and the functional relation between PS and PF guarantees that the approximation is topologically consistent.

The method can approximate both the Pareto critical set ${ }^{3}$ or the set of local Pareto optima. This can be a drawback, because there is the possibility that some globally dominated solutions to be included in the PS. Furthermore, the method is quite computationally intensive, requiring a full tessellation of the feasible set and the computation of first and second derivatives. Figures 3(b) and 4 report the workings of the SiCon algorithm on two smooth problems, where the nonlinearities and nonconvexities are responsible for the presence of multiple connected components for the PS.

\footnotetext{
3 The Pareto critical set is the set of points satisfying the first order necessary conditions for optimality (see [24])
} 


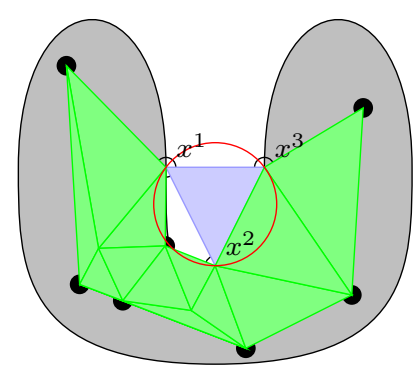

Fig. 5: The triangle with vertices $x^{1}, x^{2}$ and $x^{3}$ (drawn in blue) is in the Delaunay tessellation of the sample points (in black) but it is not in the Delaunay tessellation constrained to the set (in gray), because the center of its only circumcircle (drawn in red) is not contained in the set. The Delaunay tessellation constrained to the set (in green) clearly traces the structure of the original set.

\section{The Mathematical Basis of PAINT-SiCon Method}

The new method we propose here is an extension of the PAINT method aimed at reproducing the results of SiCon, while not being so computationally expensive, not requiring derivative information and also handling the constraints of the problem. The main issue about PAINT, which hinders consistent representations of the PS in the nonconvex case, is the missing connection between the decision space and the objective space.

As a first step, we consider the structure (i.e., the simplicial complex) emerging from the PAINT method in the objective space and then backwards propagate it on the decision space, i.e., considering a simplex having two outcomes $f\left(x^{1}\right)$ and $f\left(x^{2}\right)$ of nondominated sample points $x^{1}, x^{2} \in F$ (where $F \subset X$ is a finite sample set) as vertices one may define new simplex in the decision space having the sample points $x^{1}$ and $x^{2}$ as vertices. Similar back-propagation can be performed with simplexes of any dimension. Back-propagating a simplex is, thus, defined for the rest of the paper as forming the simplex, the vertices of which are the inverse images of the vertices of the original simplex. In this way, we can guarantee that the approximation in the decision space complies with the domination structure. However, as observed above, some of these connections can be unreasonable, therefore we need a compelling criterion for removing the invalid simplexes. We propose a criterion based on a Delaunay tessellation of the decision space. We will show that with a dense enough sampling of the feasible set, a subset of the Delaunay tessellation restricted to the feasible set $X$ can be used to extract the connected components of the PS. Then, only the simplexes that are defined by sample points in a single connected component are validated. The criterion can be generalized for constrained problems, by using restricted Delaunay tessellations to the feasible set $\left.X \subseteq \mathbb{R}^{n}[28,29]\right)$. The simplexes of a restricted tessellation have always the circumcenter inside the restricting subset. Figure 5 illustrates the difference of the Delaunay tessellation constrained to a set and the normal Delaunay tessellation.

We start with some definitions and, then, we build such tessellations characterizing the connected components of $P S$. 
Definition 1 [Simplicial Covering] Consider a simplicial complex $\mathcal{C}$. Given any $X^{\prime} \subset X$, consider the union of the simplexes in $\mathcal{C}$ intersecting $X^{\prime}$ :

$$
\mathcal{C} \cap X^{\prime}:=\bigcup\left\{S \in \mathcal{C}: S \cap X^{\prime} \neq \emptyset\right\}
$$

We refer to $\mathcal{C} \cap X^{\prime}$ as the simplicial covering of $X^{\prime}$ (based on the simplicial complex $\mathcal{C})$.

Definition 2 [Simplicial Patch] Consider a simplicial complex $\mathcal{C}$ and a set $X^{\prime} \subseteq X$. The connected components of $\mathcal{C} \cap X^{\prime}$ are referred to as the simplicial patches of the parts of $X^{\prime}$ (based on the simplicial complex $\mathcal{C}$ ).

By examining the simplicial covering of the Pareto critical set in Figure 3(b), i.e., the green triangles, one clearly notices that the simplicial patches can separate the different connected components of the PS. This is clearly a valuable feature that should be investigated more deeply. Can we say that this is true in general, or at least for a generic family of functions? More precisely we state:

Definition 3 [faithful simplicial covering] For a simplicial complex $\mathcal{C}$, a simplicial covering $\mathcal{C} \cap X^{\prime}$ of a set $X^{\prime} \subseteq X$ is called faithful if every simplicial patch contains exactly one connected component of $X^{\prime}$.

Therefore a faithful simplicial covering of a set $X^{\prime}$ is able to separate its connected components. At this point we want to prove that under suitable hypothesis on the objective functions, a simplicial covering based on the set of nondominated points is a faithful simplicial covering for PS. In order to do that we need to assume some regularity on the functions in study.

Definition 4 We say that the vector function $f: X \longrightarrow \mathbb{R}^{k}$ is sufficiently regular if

1. the function $f$ is $C^{\infty}$,

2. the PS is a stratified set of dimension $k-1^{4}$ and

3. there exists $\epsilon>0$ such that two distinct connected components of the PS are at least $\epsilon$ apart each one from the other.

Remark 1 The stratification of the global Pareto set PS, stated in point 2. of Definition 4, can be false in degenerate cases but we conjecture that in generic cases is true. The genericity of the stratification property has been proved for the local PS [26, 30, 31] but a proof for for the global PS is still missing. Also a viable analytic criterion for checking this property is not available, yet. This issue has been discussed in [20, 24-26, 32].

In addition, we are going to assume that the input points in the decision and objective spaces are in general position. Thus, all the polytopes are in fact simplexes. This can be done, because if this is not the case, then the points can be perturbed infinitesimally to guarantee general position, as is shown in [33].

Proposition 1 If $f: X \longrightarrow \mathbb{R}^{k}$ is sufficiently regular there exists a faithful simplicial covering of $P S$.

\footnotetext{
4 A stratified set can be thought as a differentiable manifold with boundary, where the boundary is a collection of differentiable manifolds of lower dimension, and the same holds for the boundary of those manifolds. See the SM for more details.
} 
Proof If $\epsilon>0$ is the minimum distance between two connected components of $P S$, then a simplicial covering where all the simplexes are strictly smaller than $\frac{\epsilon}{2}$ separates the connected components of $P S$. Indeed, consider such a simplicial covering and two points $p, q \in P S$ belonging to adjacent simplexes, i.e., to simplexes having at least a common node. Then, if the diameters of the simplexes are all smaller than $\frac{\epsilon}{2}$, we have that $|p-q|<2 \frac{\epsilon}{2}=\epsilon$. Therefore $p$ and $q$ cannot belong to different connected components of $P S$. As a result, any two points belonging to distinct connected components cannot belong to adjacent simplexes, and the covering of the $P S$ is faithful.

Since the $P S$ is not known, we need now some recipe for defining such coverings for an a priori not precisely known set $X^{\prime}$. In particular we want to know if the simplicial covering of an approximation of $X^{\prime}$ covers faithfully the unknown approximated set $X^{\prime}$. For that we define the following:

Definition 5 Given a set $X^{\prime} \subseteq X$ and a finite set of points $\Lambda=\left\{x^{1}, \ldots, x^{a}\right\}$, we say that $\Lambda$ is a $\delta$-approximation of $X^{\prime}$ if

$$
\max \left(\max _{x \in X^{\prime}} \min _{y \in \Lambda} d(x, y), \max _{y \in \Lambda} \min _{x \in X^{\prime}} d(x, y)\right)=: d_{\mathcal{H}}\left(X^{\prime}, \Lambda\right) \leqslant \delta,
$$

i.e., $d_{\mathcal{H}}\left(X^{\prime}, \Lambda\right)$ is the Hausdorff distance between the sets $X^{\prime}$ and $\Lambda$.

With the above definition we can give sufficient conditions for a simplicial covering to be faithful. This is given by the following theorem.

Theorem 1 Let $X^{\prime} \subseteq X$ and let $\delta>0$ be the minimum Hausdorff distance between two connected components of $X^{\prime}$. Let $\Lambda$ be a sample of points where the maximum diameter of an empty ball is $\delta^{\prime}>0$, and let $\mathcal{D}$ be a Delaunay tessellation based on $\Lambda$. Let $F \subseteq \Lambda$ be the points at a distance smaller than $\delta^{\prime}$ from a point of $X^{\prime}$. Then $F$ is a $\delta^{\prime}$-approximation of $X^{\prime}$ and the subset of simplexes of $\mathcal{D}$ having all vertices in $F$ is a simplicial covering of $X^{\prime}$. If $\delta^{\prime}<\frac{\delta}{2}$, then the simplicial covering is also faithful for $X^{\prime}$.

Proof The proof can be divided in the following steps:

1. The maximum diameter of the Delaunay tessellation cells is smaller or equal than $\delta^{\prime}$.

2. $F$ is actually a $\delta^{\prime}$-approximation of $X^{\prime}$. Indeed it is clear that for each point $s \in X^{\prime}$ there exists a point $v$ in $\Lambda$, and therefore in $F$, at a distance smaller that $\delta^{\prime}$ from $s$. Assume by absurdum that for a point $s \in X^{\prime}$ there is no point of $\Lambda$ in a ball of radius $\delta^{\prime}$. The existence of a ball with radius $\delta^{\prime}$ not intersecting $\Lambda$ is in contradiction with the hypothesis on $\Lambda$.

3. If a simplex $\tau$ in the tessellation intersects $X$, then its vertices are in $X^{\prime}$. Indeed every point in $\tau$ is within a $\delta^{\prime}$ distance from each of its vertices. Therefore, $\mathcal{C}$, i.e., the set of simplexes with all vertices in $X^{\prime}$, is a simplicial covering of $X$.

4. Consider two points from two distinct connected components in $X, s$ and $q$. Their respective distance is larger than $\delta$. If there exists two adjacent simplexes, one containing $s$ and one containing $q$, there should exist at least a common vertex $v$ for two simplexes, with $v \in X^{\prime}$. But for the triangle inequality it should be

$$
0<\delta<d(s, q) \leqslant d(s, v)+d(v, q) \leqslant \delta^{\prime}+\delta^{\prime},
$$

which cannot hold if $\delta>2 \delta^{\prime}$. Therefore $\mathcal{C}$ is a faithful simplicial covering of $X$. 
On the basis of Theorem 1, we claim that the connected components of the PS of a generic objective vector function can be distinguished consistently by a simplicial covering based on the nondominated set of a sufficiently fine approximation.

Theorem 2 Let $G$ be a grid of points in $X$ with maximum diameter of an empty ball equal to $\delta$, and let $F$ be the subset of nondominated points. If for every Pareto optimal solution there is a nondominated point in $F$ at a distance smaller than $\delta>0$, and the minimum separation distance among two connected components of $P S$ is larger than $5 \delta$, then the set of Delaunay simplexes having at least a node in $F$ is a faithful simplicial covering of $P S$.

Proof Although, we assume that every point in $P S$ can be approximated by a nondominated point in the sample $G$, i.e., by a point in $F$, we cannot say that all points of $G$ in a $\delta$-neighborhood of $\mathcal{P S}$ are nondominated. In practice this is what one observes, e.g., Figure 7 . So $F$ is not a $\delta$-approximation of $P S$, however, in our hypothesis, we have that every point in $P S$ is contained in a simplex with at least a vertex in $F$. Therefore, if the connected components of $P S$ are distant at least $4 \delta$, we have that the simplicial covering $\mathcal{C}$ composed by the simplexes having at least one vertex in $F$ is faithful for $P S$.

Indeed, simplicial covering $\mathcal{C}$ must be contained in a tubular neighborhood of $P S$ of radius $2 \delta^{5}$. Indeed, $\mathcal{C}$ is composed by simplexes intersecting $P S$ and some of the empty simplexes sharing at least one vertex with a nonempty simplex. If the sizes of those simplexes is smaller than $\delta$, the farthest point from $P S$ is at a distance smaller than $2 \delta$. Therefore, the tubular neighborhoods of distinct connected components cannot intersect, and the covering is faithful.

Remark 2 Thus, by building a Delaunay tessellation of the decision space and selecting the simplexes having at least a nondominated vertex, we have a correct representation of the connected components of the PS. Therefore, we could consider the simplexes defined by the PAINT method and validate only those contained in the simplicial patches of the covering. However, this strategy requires the full computation of the Delaunay tessellation, which is likely to be exhaustive in the interesting cases. Thus, we propose to detect the connected components by defining a graph which nodes are the nondominated points and which line segments (also called links and 1-dimensional polytopes, in this context) are Delaunay polytopes. This structure is considerably less expensive than the construction of the full tessellation. At this point, if one validates only the Delaunay polytopes among the PAINT simplexes, practical experience suggests that a great number of valid simplexes would be rejected. We propose then to accept all PAINT simplexes whose extrema belong to the same connected components. This is illustrated more in detail in the next section.

\section{The PAINT-SiCon Method}

The input of the PAINT-SiCon algorithm is a sampling $\Lambda$ of the feasible set $X$ and, if the sampling is fine enough and the functions are sufficiently regular, the

\footnotetext{
5 A tubular neighborhood of a set $S \subset X$ with radius $r>0$ is defined as $T_{S, r}:=$ $\{x \in X: d(x, S)<r\}$
} 
algorithm outputs a PS approximation which is a simplicial complex and separates the connected components. We start by evaluating the objective functions on a given set of points, then we extract the nondominated set and build the approximation of the PS according to the PAINT method. We then back-propagate the approximation in to the decision space and validate the back-propagated simplexes by checking whether the vertices of the link are in the set of vertices of the same simplicial patch based on the Delaunay complex restricted to the feasible set $S$. The method described above is computationally less expensive than the SiCon method, but the computational burden is still not entirely negligible, if we require to compute the full Delaunay tessellation in the decision space. This difficulty can be overcome by checking the Delaunay condition only for the links we are interested. This choice considerably simplifies the procedure and makes the method efficient and appealing. Algorithm 1 describes the functionality of the PAINT method in more detail. Algorithm 2 partitions the Delaunay tessellation

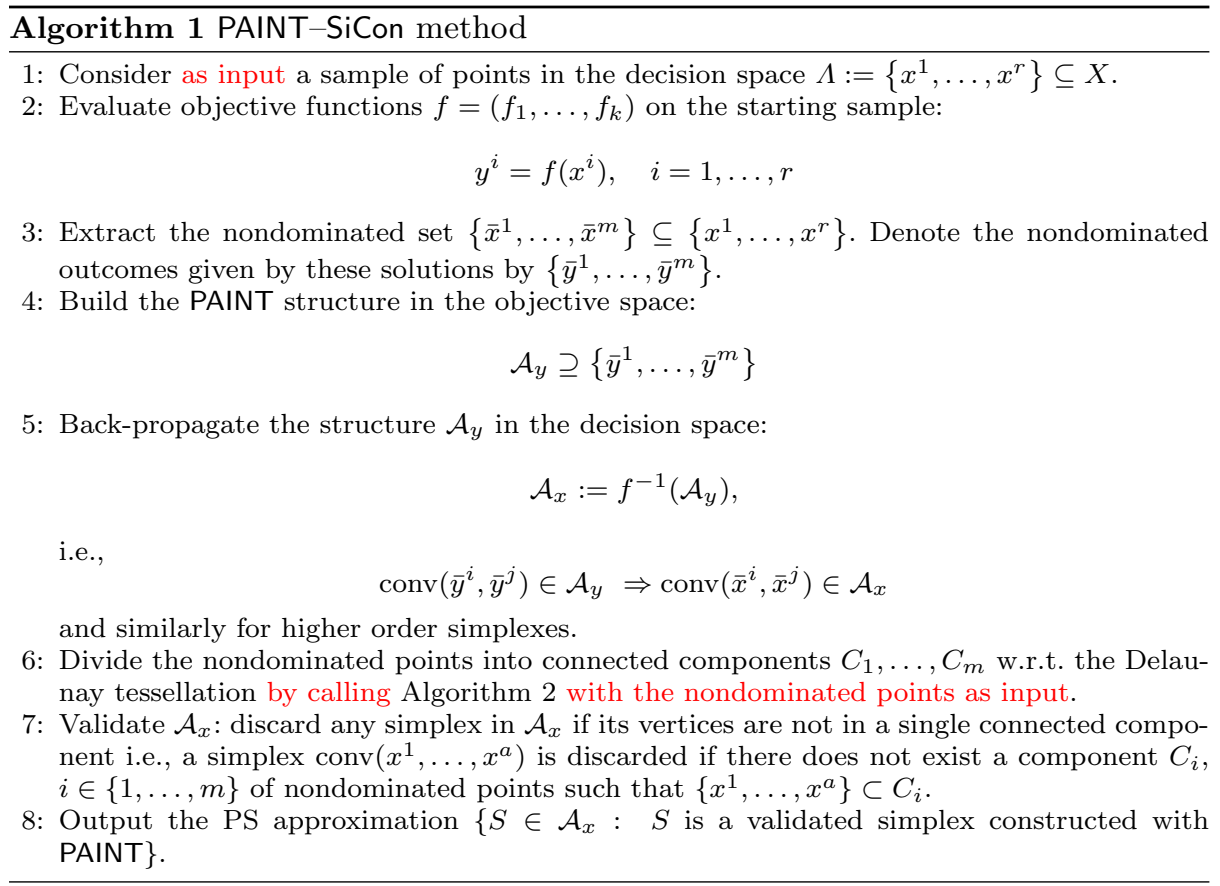

defined on the nondominated set $F$ into connected components. A straightforward way to do step 1. of Algorithm 2 is to construct the Delaunay tessellation of the complete sample set $\Lambda$ restricted to the feasible set $S$ and then considering only the 1-dimensional simplexes. After this, one can check whether both of the end points are nondominated points and, if so, include the simplex in $\mathcal{D}_{X}^{1, \Lambda}$. However, this approach is computationally expensive, as only the complexity of computing the restricted Delaunay tessellation in the decision space is exponential in the number of dimensions. 


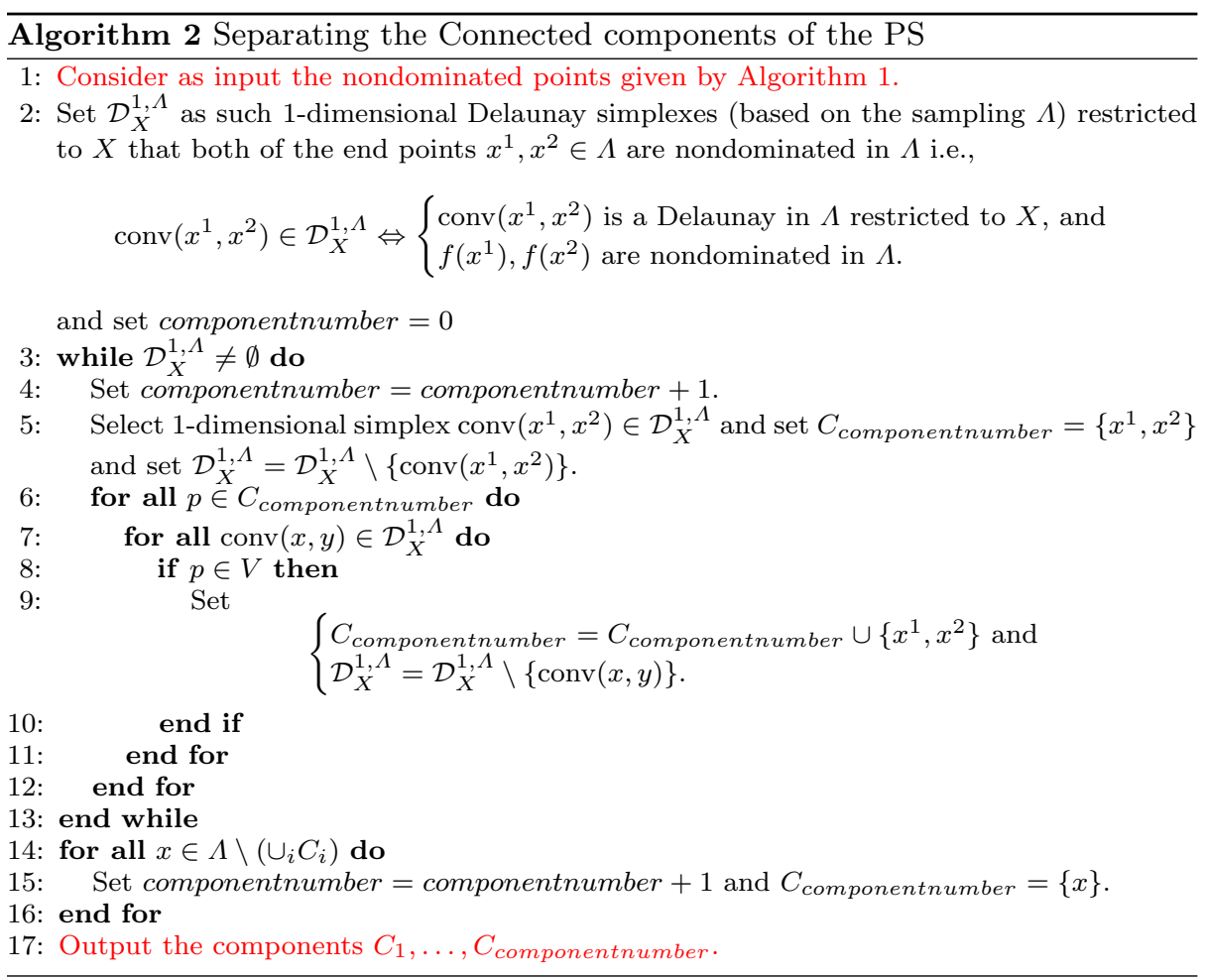

We have, however, developed a new optimization-based approach to finding the 1-dimensional Delaunay simplexes, which are needed for Algorithm 2. By definition, a simplex $\operatorname{conv}\left(x^{1}, x^{2}\right)$ belongs to the restricted Delaunay tessellation [28] if and only if there exists an open ball $B(x, r)$ such that the center $x \in X$, $\bar{B}(x, r) \cap \Lambda=\left\{x^{1}, x^{2}\right\}$ and $B(x, r) \cap \Lambda=\emptyset$. Since the feasible set is often described in multiobjective optimization problems by mathematical constraints, a natural way of checking whether the simplex is in this Delaunay tesselation is by solving the optimization problem

$$
\begin{array}{ll}
\max & t \\
\text { s.t. } & \left\|x^{1}-x\right\|=\left\|x^{2}-x\right\| \\
& \|y-x\| \geqslant\left\|x^{1}-x\right\|+t \text { for all } y \in \Lambda \backslash\left\{x^{1}, x^{2}\right\} \\
& x \in X \text { and } t \in \mathbb{R} .
\end{array}
$$

If the optimal value of the problem (5) is greater than zero, then the simplex defined by these points is Delaunay; otherwise, it is not Delaunay. The problem can be written as

$$
\begin{array}{ll}
\max & t \\
\text { s.t. } & \sum_{i=1, \ldots, n}\left(\left(x_{i}^{2}\right)^{2}-2 x_{i}^{2} x^{i}\right)=\sum_{i=1, \ldots, n}\left(\left(x_{i}^{1}\right)^{2}-2 x_{i}^{1} x^{i}\right) \\
& \sum_{i=1, \ldots, n}\left(\left(y_{i}\right)^{2}-2 y_{i} x^{i}\right) \geqslant \sum_{i=1, \ldots, n}\left(\left(x_{i}^{1}\right)^{2}-2 x_{i}^{1} x^{i}\right)+t \\
& \quad \text { for all } y \in \Lambda \backslash\left\{x^{1}, x^{2}\right\} \\
& x \in X \text { and } t \in \mathbb{R},
\end{array}
$$


which is linear if the constraints setting $X$ are linear. Thus, one needs to solve $a(a-1)$ (where $a$ is the number of nondominated points in the sample $F$ ) times problem (6) for finding out the restricted Delaunay 1-dimensional simplexes of nondominated points in the sample. If the constraints are linear, the optimization problems can be solved with interior point methods in polynomial time [34]. Even if the case of nonlinear constraints, this approach still saves the computational cost of checking the Delaunay property.

After the above, the algorithm starts with a random simplex in the collection $\mathcal{D}_{X}^{\prime}$ and first sets that the vertices of this 1-dimensional simplex are in this component. Then, the algorithm starts inspecting which sample points are connectable (using the 1-dimensional simplexes in $\mathcal{D}_{X}^{\prime}$ ) from each of the sample points already in the sample. The whole component has been found, when there are no other sample points that are connected to sample points in the component with 1-dimensional simplexes in $\mathcal{D}^{\prime}$. Finally, the algorithm sets the nondominated sample points that are not connected to any other sample points as components that have merely one sample point.

Figure 6 shows the Delaunay 1-dimensional simplexes of of nondominated points (the labeled points) for an arbitrarily defined sample set. In this example, the collection $\mathcal{D}_{X}^{\prime}$ would thus contain 1-dimensional simplexes $\operatorname{conv}\left(x^{5}, x^{14}\right)$, $\operatorname{conv}\left(x^{14}, x^{4}\right), \operatorname{conv}\left(x^{4}, x^{5}\right), \operatorname{conv}\left(x^{16}, x^{25}\right)$ and so on. Thus, the first component would by the first 1-dimensional simplex contain points $x^{5}, x^{14}$. In addition, because the third 1-dimensional simplex contains $x^{5}$, the point $x^{15}$ would also be in this component. No other points would be in the first component, because only the second 1-dimensional simplex contains the point $x^{14}$, but the sample point $x^{15}$ is already in the component. Thus, the first component Component Com $_{1}=\left\{x^{5}, x^{14}, x^{15}\right\}$. In a similar way, this algorithm concludes that Component Com $_{2}=\left\{x^{16}, x^{24}, x^{25}\right\}$ and Component $_{3}=\left\{x^{31}, x^{30}, x^{38}, x^{37}\right\}$.

Once the PAINT-SiCon approximation $\mathcal{A}$ has been constructed, it can be represented with almost the same surrogate problem as the PAINT surrogate. Let each simplex $S \in \mathcal{A}$ be represented as a row $A_{i}$ in a matrix $A$ so that $S=$ $\operatorname{conv}\left(x^{A_{i, 1}}, \ldots, x^{A_{i, b}}\right)$, where $x^{i}$ are solutions in the sample $X^{\prime}$ and $b \in \mathbb{N}$ is an integer. With this matrix, the PAINT-SiCon surrogate problem can be written as

$$
\begin{array}{ll}
\max & \left(z_{1}, \ldots, z_{k}\right) \\
\text { s.t. } & \sum_{j=1}^{a} \sum_{l=1}^{b} \lambda_{j, l}=1 \\
& \sum_{l=1}^{b} \lambda_{j, l} \leqslant y_{j}, \text { for all } j=1, \ldots, a \\
& \sum_{j=1}^{b} y_{j}=1 \\
& \lambda \in[0,1]^{a \times b} \\
& y \in\{0,1\}^{a}, \\
& z_{i}=\sum_{j=1}^{a} \sum_{l=1}^{b} \lambda_{j, l} f_{i}\left(x^{A_{l, j}}\right) \text { for all } i=1, \ldots, k \text { and } \\
& x=\sum_{j=1}^{a} \sum_{l=1}^{b} \lambda_{j, l} x^{A_{l, j}} .
\end{array}
$$

In the above problem also the Pareto optimal solutions $x$ are approximated and not only the Pareto optimal outcomes $z$. Thus, the decision maker does not have to find the corresponding solution to the actual problem to get information about the values of the decision variables. In addition, because the PAINT-SiCon method validates the polytopes in the decision space, the feasible values of the variable $x$ in Problem (7) does not contain values that are far away from the connected 


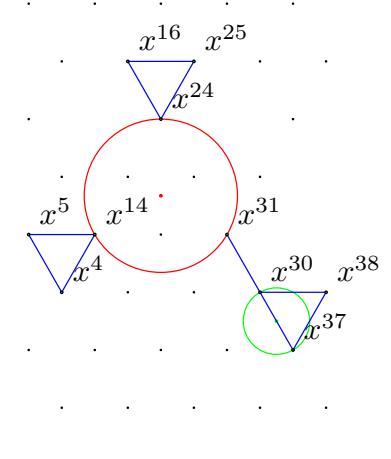

(a)

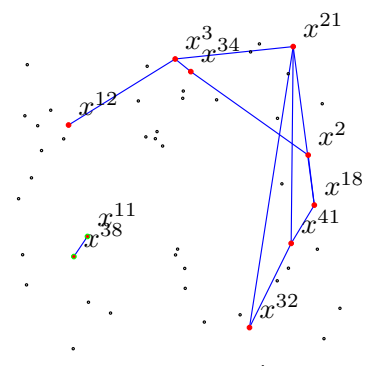

(b)

Fig. 6: (a) The valid unrestricted Delaunay 1-dimensional simplexes of nondominated points (the labeled points) in a 2-dimensional decision space are marked with blue color. The circumscribing circle (and its center) of simplex $\operatorname{conv}\left(x^{14}, x^{24}\right)$, where the difference of the distance to sample points $x^{14}$ and $x^{24}$ and the distance to other sample points is the biggest is drawn in red color. The fact that there are sample points inside this circle shows that the simplex $\operatorname{conv}\left(x^{14}, x^{24}\right)$ is not Delaunay. On the other hand, the circumscribing circle for the Delaunay simplex $\operatorname{conv}\left(x^{37}, x^{30}\right)$ drawn in green does not contain any sample points and this shows that the simplex is indeed Delaunay. (b) The valid Delaunay simplexes of nondominated points (the labeled points) in a 3-dimensional decision space are marked with blue color. In this example, there are 2 components; the sample points in one component are marked with green color and those of the other component are marked with red color.

components of the PS, and, because of continuity, the values of the objective functions are accurately approximated by the linearly approximated values given by the PAINT-SiCon surrogate problem.

\section{Examples}

In this section, examples of applying the PAINT-SiCon method are shown. Our test problems are the two-objective $L \& H_{2 \times 2}$ with two-dimensional decision space and the three-objective $L \& H_{3 \times 3}$ with three-dimensional decision space that have both been developed by A. Lovison. In both problems there is a PS composed by two distinct connected components, which give rise to two local PFs intersecting each other. For testing the algorithm, we have implemented it using the Octave [35] scripting language. For the sake of clarity, we give the explicit construction of these problems, which could be useful in order to construct problems with similar features. Basically we combine smooth bump functions, defined as Gaussians, and operate projections and rotations for orienting the fronts in the desired directions. $L \& H_{2 \times 2}$ is defined by summing two Gaussians, one centered in $(0,0)$, the other in $(0,-1.5)$, the first bump with a larger amplitude, the second appreciably higher and sharper. The graph of the sum is projected on the $(x, z)$ plane, such that the axes of the bumps are projected one above the other. Finally the plane is rotated by 45 degrees such that the axes of the gaussians become oriented as the vector 
$(1,1)$. More formally:

$$
\begin{array}{ll}
\max & L \& H_{2 \times 2}(x, y) \\
\text { s.t. } & -0.75 \leqslant x \leqslant 0.75, \\
& -2.5 \leqslant y \leqslant 0.12,
\end{array}
$$

where

$$
\begin{gathered}
\operatorname{gau}\left(p, p_{0}, \sigma\right):=\sqrt{\frac{2 \pi}{\sigma}} \exp \left(-\frac{\left|p-p_{0}\right|^{2}}{\sigma^{2}}\right), \\
p_{L}=(0 ., 0 .)^{\top}, \quad p_{H}=(0 .,-1.5)^{\top}, \\
\left.\operatorname{bumps}(p):=0.2 \times \operatorname{gau}\left(p, p_{L}\right), 0.65\right)+1.5 \times \operatorname{gau}\left(p, p_{H}, 2.8\right), \\
L \& H_{2 \times 2}(x, y):=\left\{\begin{array}{l}
f_{1}(x, y):=\frac{\sqrt{2}}{2} x+\frac{\sqrt{2}}{2} \operatorname{bumps}\left((x, y)^{\top}\right), \\
f_{2}(x, y):=-\frac{\sqrt{2}}{2} x+\frac{\sqrt{2}}{2} \operatorname{bumps}\left((x, y)^{\top}\right) .
\end{array}\right.
\end{gathered}
$$

This function is smooth (is $C^{\infty}$ ), and from the construction it is clear that the PS is composed by three smooth curved intervals (i.e., $k-1$ submanifolds with boundary, with $k=2){ }^{6}$ Then the function is sufficiently regular. As claimed in Theorems 1 and $2 a$ sufficiently fine grid will produce faithful simplicial coverings.

The $L \& H_{3 \times 3}$ problem is a generalization to three dimensions of the previous one. The PS of this problem also consists of two connected components, which for three objectives is a 2 dimensional stratified set $[24,25,36]$. This problem can be formulated as

$$
\begin{array}{ll}
\max & L \& H_{3 \times 3}(x, y, z), \\
\text { s.t. } & -0.5 \leqslant x \leqslant 0.5, \\
& -1.5 \leqslant y \leqslant 0.5, \\
& -0.5 \leqslant z \leqslant 0.5,
\end{array}
$$

where

$$
\begin{gathered}
\operatorname{gau}\left(p, p_{0}, \sigma\right):=\sqrt{\frac{2 \pi}{\sigma}} \exp \left(-\frac{\left|p-p_{0}\right|^{2}}{\sigma^{2}}\right) \\
p_{L}=(0,0.15,0)^{\top}, \quad p_{H}=(0,-1.1,0)^{\top}, \\
\left.\operatorname{bumps}(p):=0.075 \times \operatorname{gau}\left(p, p_{L}\right), 0.3\right)+1 . \times \operatorname{gau}\left(p, p_{H}, 3 .\right) .
\end{gathered}
$$

Let us consider the rotation of the space around the vector $\omega$ of an angle $\theta$ :

$$
\rho(p):=\operatorname{Rot}(p, \omega, \theta), \quad \omega:=\left(-\frac{\sqrt{2}}{2}, \frac{\sqrt{2}}{2}, 0\right)^{\top}, \quad \theta=\arctan (\sqrt{2}),
$$

notice that this rotation brings the vector $(0,0,1)^{\top}$ on the vector $\frac{(1,1,1)^{\top}}{\sqrt{3}}$. Finally we set:

$$
L \& H_{3 \times 3}(x, y, z):=\rho((x, z, \operatorname{bumps}(x, y, z))) .
$$

Also $L \& H_{3 \times 3}$ is smooth and its PS is formed by two smooth 2-dimensional submanifolds, whose boundary is composed by smooth curved intervals. ${ }^{7}$ Therefore the function is sufficiently smooth and according to Theorems 1 and 2 a fine grid will separate consistently the components.

\footnotetext{
6 See also Figure 3 (b)).

7 See also Figure 4.
} 


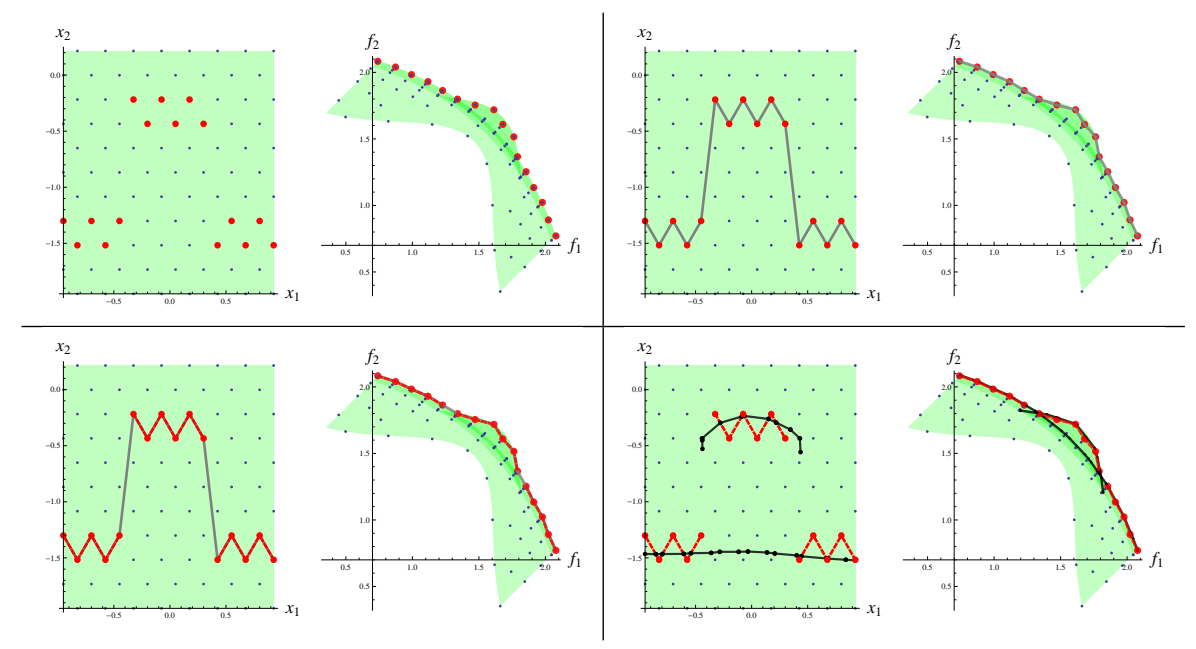

Fig. 7: Application of PAINT-SiCon on the $L \& H_{2 \times 2}$ problem.(Top-Left): a sample of points with the corresponding objectives values. The red points denote the nondominated set. (TopRight): the gray lines represent the output of PAINT. (Bottom-Left): red lines represent the structure validated via PAINT-SiCon. (Bottom-Right): output of PAINT-SiCon (red lines) compared with the output of SiCon (black lines).

Figure 7 demonstrates the application of the PAINT-SiCon method to approximating the PS of the $L \& H_{2 \times 2}$ problem. The top panel in the figure shows the input data with the nondominated solutions and outcomes highlighted. The second panel from the top shows the PAINT simplexes and the simplexes propagate into the decision space. In the second panel from the bottom, the simplexes that have been validated are highlighted. Finally, the bottom figure compares the results of the PAINT-SiCon algorithm (red zigzag pattern) and the SiCon algorithm (black lines). It can be seen that the results are qualitatively similar, but we notice that PAINT-SiCon did not made use of derivative information and did not require the computation of the full Delaunay tessellation of the decision space. Figure 8 illustrates the application of the PAINT-SiCon method to the $L \& H_{3 \times 3}$ problem. The top panels in Figure 8 show the back-propagated PAINT simplexes (on the left) and the PAINT simplexes in the objective space (on the right). The bottom panels in the figure show the PAINT simplexes that have been validated both in the decision space (on the left) and in the objective space (on the right). For this problem also, the PAINT-SiCon method has correctly approximated the shape of the PF i.e., the approximation also consists of two connected components.

Surrogate problem (7) for this multiobjective optimization problem have parameter $a=13$ describing the simplexes and $b=2$ describing the maximal number of vertices in a simplex ${ }^{8}$. The matrix $A$ characterizes the simplexes in a way described in Section 2.2 e.g., when the first simplex has as its vertices solutions $x^{17}$ and $x^{18}$ then the first line of the matrix $A$ is $[17,18]$. Problem $(7)$ is a mixed-integer linear multiobjective optimization problem and it can now be used as a surrogate

\footnotetext{
8 Note that, all the simplexes are now line segments.
} 

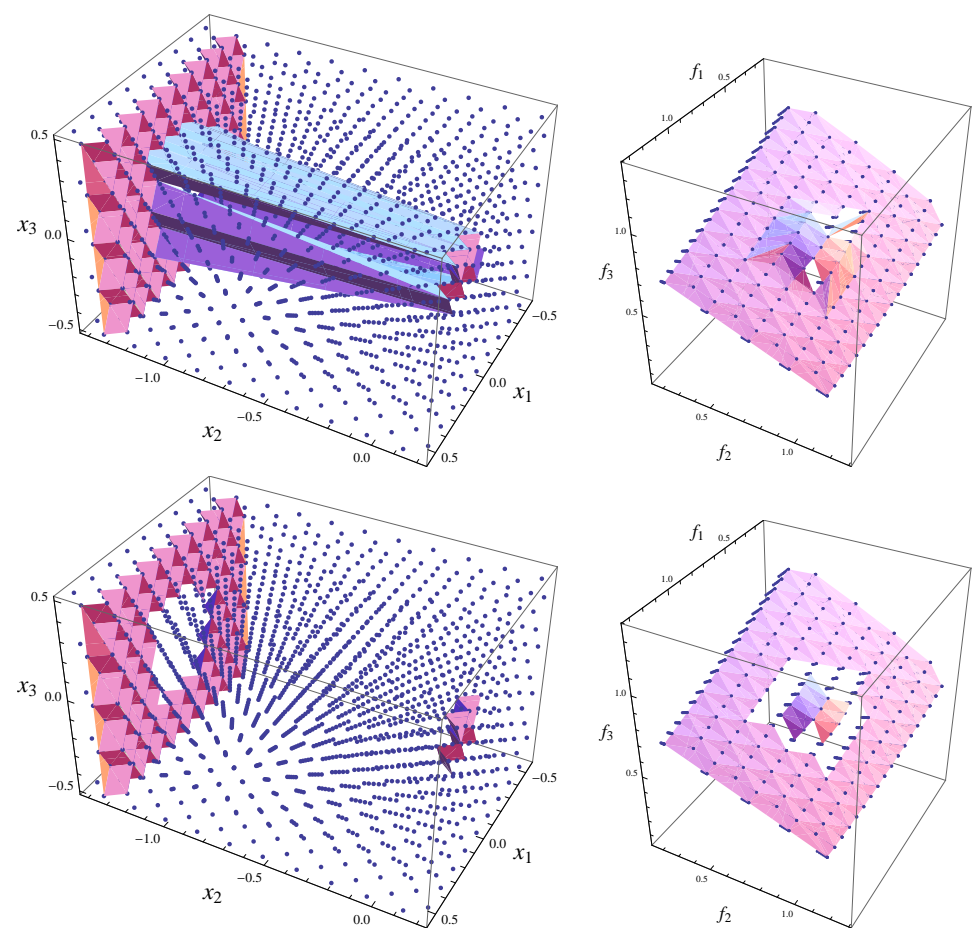

Fig. 8: Application of PAINT-SiCon on the $L \& H_{3 \times 3}$ problem. (Top): output of the PAINT method. (Bottom): selection of the consistent simplexes according to the PAINT-SiCon method. For comparison with the SiCon method, see figure 4.

of the original problem in e.g., interactive decision making or any a posteriori multiobjective optimization method.

\section{Conclusions and Topics of Further Research}

In this paper, we have introduced the PAINT-SiCon PS approximation method that is an extension of the PAINT method using the ideas of the SiCon method. The method produces consistent parametric representations of the PS and the PF based on a sampling in the feasible set. These parametric representations can be used as a computationally inexpensive surrogate for the original multiobjective optimization problem. The method has been especially developed for nonconvex problems and can detect separate connected components of the PF unlike the PAINTmethod. In addition, the PAINT-SiCon method is computationally less expensive than the SiCon method.

As a topic of further research, great interest is in the definition of refinement strategies. The question remains if one can utilize the PS approximation constructed by the PAINT-SiCon method to produce new Pareto optimal solutions without building explicitly the Delaunay tessellation of the feasible set. Clearly, 
the points near the PS approximation are natural candidates for improving the approximation. Producing well distributed candidate points in the neighborhoods of the PAINT-SiCon structure, leading to more and more accurate approximations of the PS is still an open problem. Another open question is how to prevent getting stagnated in the neighborhoods of the first approximation detected, possibly missing a global branch of Pareto optimal points located in a still unexplored region of the feasible set. Clearly this question is a multiobjective version of the exploration-exploitation dilemma of global optimization.

Finally, when the method is used on real world applications, checking the smoothness conditions on such functions is usually impracticable. However, as noticed above, these conditions are not restrictive and we can expect that they are generally met, at least after an arbitrarily small perturbation.

\section{References}

1. C. Hwang and A. S. M. Masud. Multiple Objective Decision Making - Methods and Applications: a State-of- the-Art Survey. Springer-Verlag Berlin, Heidelberg, 1979 .

2. K. Miettinen. Nonlinear Multiobjective Optimization. Kluwer Academic Publishers, Boston, 1999.

3. V. Pareto. Manuale di economia politica con una introduzione alla scienza sociale. Piccola biblioteca scientifica. Società editrice libraria, Milan, 1906.

4. H. P. Benson and S. Sayin. Towards Finding Global Representations of the Efficient Set in Multiple Objective Mathematical Programming. Naval Research Logistics, 44:47-67, 1997.

5. E. Karasakal and M. Köksalan. Generating a Representative Subset of the Nondominated Frontier in Multiple Criteria Decision Making. Operations Research, 57:187-199, 2009.

6. K. Klamroth and K. Miettinen. Integrating Approximation and Interactive Decision Making in Multicriteria Optimization. Operations Research, 56:222234, 2008.

7. E. Noor and R. Milo. Efficiency in Evolutionary Trade-Offs. Science, 336:11141115, May 2012.

8. O. Shoval, H. Sheftel, G. Shinar, Y. Hart, O. Ramote, A. Mayo, E. Dekel, K. Kavanagh, and U. Alon. Evolutionary Trade-Offs, Pareto Optimality, and the Geometry of Phenotype Space. Science, 336:1157-1160, May 2012.

9. S. Ruzika and M. M. Wiecek. Approximation Methods in Multiobjective Programming. Journal of Optimization Theory and Applications, 126:473-501, 2005.

10. C Hillermeier. Generalized Homotopy Approach to Multiobjective Optimization. Journal of Optimization Theory and Applications, 110:557-583, 2001.

11. C. Hillermeier. Nonlinear Multiobjective Optimization: a Generalized Homotopy Approach. Birkhäuser Verlag, Basel, 2001.

12. O Schütze, A. Dell'Aere, and M. Dellnitz. On Continuation Methods for the Numerical Treatment of Multi-Objective Optimization Problems. In Practical Approaches to Multi-Objective Optimization. IBFI, Schloss Dagstuhl, Germany, 2005. 
13. M. Hartikainen, K. Miettinen, and M. M. Wiecek. Constructing a Pareto Front Approximation for Decision Making. Mathematical Methods of Operations Research, 73:209-234, 2011.

14. M. Hartikainen, K. Miettinen, and M. M. Wiecek. Pareto Front Approximations for Decision Making with Inherent Non-dominance. In New State of MCDM in the 21st Century, pages 35-46. Springer-Verlag, 2011.

15. S. Askar and A. Tiwari. Multi-Objective Optimisation Problems: A Symbolic Algorithm for Performance Measurement of Evolutionary Computing Techniques. In M. Ehrgott, editor, EMO 2009, pages 169-182. Springer-Verlag, 2009.

16. I. Das and J. E. Dennis. Normal-Boundary Intersection: a New Method for Generating the Pareto Surface in Nonlinear Multicriteria Optimization Problems. SIAM Journal on Optimization, 8:631-657, 1998.

17. J. Rakowska, R. T. Haftka, and L. T. Watson. Multi-Objective ControlStructure Optimization via Homotopy Methods. SIAM Journal on Optimization, 3:654-667, 1993.

18. B. Grünbaum. Convex Polytopes. Interscience Publishers, London, 1967.

19. A. Lovison. Singular Continuation: Generating Piecewise Linear Approximations to Pareto Sets via Global Analysis. SIAM Journal on Optimization, 21:463-490, 2011.

20. A. Lovison. Global Search Perspectives for Multiobjective Optimization. Journal of Global Optimization, 57(2):385-398, 2013.

21. M. Hartikainen, K. Miettinen, and M. M. Wiecek. PAINT: Pareto Front Interpolation for Nonlinear Multiobjective Optimization. Computational Optimization and Applications, 52:845-867, 2012.

22. K. Deb, L. Thiele, M. Laumanns, and E. Zitzler. Scalable Multi-Objective Optimization Test Problems. IEEE International Conference on E-Commerce Technology, 1:825-830, 2002.

23. A. P. Wierzbicki. On the Completeness and Constructiveness of Parametric Characterizations to Vector Optimization Problems. OR Spectrum, 8:73-87, 1986.

24. S. Smale. Global Analysis and Economics. I. Pareto Optimum and a Generalization of Morse Theory. In Dynamical systems (Proc. Sympos., Univ. Bahia, Salvador, 1971), pages 531-544. Academic Press, New York, 1973.

25. W. de Melo. Stability and Optimization of Several Functions. Topology, 15:1$12,1976$.

26. Y. H. Wan. On the Algebraic Criteria for Local Pareto Optima. I. Topology, 16:113-117, 1977.

27. E. L. Allgower and K. Georg. Piecewise Linear Methods for Nonlinear Equations and Optimization. Journal of Computational and Applied Mathematics, 124:245-261, Jan 2000.

28. H. Edelsbrunner. Shape Reconstruction with Delaunay Complex. In C. L. Lucchesi and A. V. Moura, editors, LATIN'98: Theoretical Informatics, pages 119-132. Springer-Verlag Berlin, Heidelberg, 1998.

29. J. E. Goodman and J. O'Rourke, editors. Discrete and Computational Geometry. CRC Press, Boca Raton, 1997.

30. W. de Melo. On the Structure of the Pareto Set of Generic Mappings. Boletim da Sociedade Brasileira de Matemática, 7:121-126, 1976. 
31. A. Lovison and F. Pecci. Hierarchical stratification of Pareto sets. Submitted, 2014.

32. R. Thom. Les singularités des Applications Différentiables. Annales de l'institut Fourier, 6:43-87, 1956.

33. H. Edelsbrunner and E. P. Mücke. Simulation of Simplicity: a Technique to Cope with Degenerate Cases in Geometric Algorithms. ACM Transactions on Graphics, 9:66-104, 1990.

34. K. Anstreicher. Linear Programming in $\mathrm{O}([\mathrm{n} 3 / \mathrm{ln} \mathrm{n}] \mathrm{L})$ Operations. SIAM Journal on Optimization, 9:803-812, 1999.

35. J. W. Eaton. GNU Octave Manual. Network Theory Limited, 2002.

36. J. Mather. Notes on topological stability. Bulleting of the American Mathematical Society (New Series), 49:475-506, 2012. 